Louisiana State University

LSU Digital Commons

Faculty Publications

Department of Biological Sciences

$5-1-2017$

\title{
Control of DNA end resection by yeast Hmo1p affects efficiency of DNA end-joining
}

Arvind Panday

Louisiana State University

Li Juan Xiao

Louisiana State University

Ashish Gupta

Louisiana State University

Anne Grove

Louisiana State University

Follow this and additional works at: https://digitalcommons.Isu.edu/biosci_pubs

\section{Recommended Citation}

Panday, A., Xiao, L., Gupta, A., \& Grove, A. (2017). Control of DNA end resection by yeast Hmo1p affects efficiency of DNA end-joining. DNA Repair, 53, 15-23. https://doi.org/10.1016/j.dnarep.2017.03.002

This Article is brought to you for free and open access by the Department of Biological Sciences at LSU Digital Commons. It has been accepted for inclusion in Faculty Publications by an authorized administrator of LSU Digital Commons. For more information, please contact ir@lsu.edu. 


\title{
Control of DNA end resection by yeast Hmo1p affects efficiency of DNA end-joining
}

\author{
Arvind Panday ${ }^{1}$, LiJuan Xiao ${ }^{2}$, Ashish Gupta, Anne Grove* \\ Department of Biological Sciences, Louisiana State University, Baton Rouge, LA 70803, USA
}

\section{A R T I C L E I N F O}

\section{Article history:}

Received 9 November 2016

Received in revised form 3 January 2017

Accepted 7 March 2017

Available online 9 March 2017

\section{Keywords:}

High mobility group protein

DNA end resection

Alternate end-joining

Plasmid end-joining

NHEJ

\begin{abstract}
A B S T R A C T
The primary pathways for DNA double strand break(DSB) repair are homologous recombination (HR) and non-homologous end-joining (NHEJ). The choice between HR and NHEJ is influenced by the extent of DNA end resection, as extensive resection is required for HR but repressive to NHEJ. Conversely, association of the DNA end-binding protein $\mathrm{Ku}$, which is integral to classical NHEJ, inhibits resection. In absence of key NHEJ components, a third repair pathway is exposed; this alternative-end joining (A-EJ) is a highly error-prone process that uses micro-homologies at the breakpoints and is initiated by DNA end resection. In Saccharomyces cerevisiae, the high mobility group protein Hmo1p has been implicated in controlling DNA end resection, suggesting its potential role in repair pathway choice. Using a plasmid end-joining assay, we show here that absence of Hmo1p results in reduced repair efficiency and accuracy, indicating that Hmo1p promotes end-joining; this effect is only observed on DNA with protruding ends. Notably, inhibition of DNA end resection in an hmo1 $\Delta$ strain restores repair efficiency to the levels observed in wild-type cells. In absence of Ku, HMO1 deletion also reduces repair efficiency further, while inhibition of resection restores repair efficiency to the levels observed in $k u \Delta$. We suggest that Hmo1p functions to control DNA end resection, thereby preventing error-prone A-EJ repair and directing repairs towards classical NHEJ. The very low efficiency of DSB repair in $k u \Delta h m o 1 \Delta$ cells further suggests that excessive DNA resection is inhibitory for A-EJ.
\end{abstract}

(c) 2017 Elsevier B.V. All rights reserved.

\section{Introduction}

DNA double strand breaks (DSBs) are cytotoxic lesions that may be caused either by exogenous factors such as ionizing radiation or as a result of metabolic processes, including DNA replication. Persistent DSBs threaten genomic integrity and may induce chromosomal rearrangements and lead to cell death or tumorigenesis. By contrast, programmed DSBs, such as those involved in meiotic recombination and in $\mathrm{V}(\mathrm{D}) \mathrm{J}$ recombination are critical for genetic diversity and for lymphogenesis $[1,2]$.

Regardless of origin, DSBs are repaired by distinct pathways that are conserved from yeast to humans. Since DSBs occur in the context of chromatin, repair pathway choice is preceded by chromatin remodeling events that allow access to repair machineries [3]. Homologous recombination (HR) requires the presence of an

\footnotetext{
* Corresponding author.

E-mail address: agrove@lsu.edu (A. Grove).

1 Current address: Department of Molecular Biosciences, University of Texas, Austin.

2 Current address: Labcorp, Phoenix, AZ.
}

intact homologous donor sequence that is used as a template to repair the chromosomal break. HR is initiated by $5^{\prime}$ to $3^{\prime}$ resection of one DNA strand to generate a $3^{\prime}$-single-stranded segment that searches for homology within the template duplex after associating with Rad51. In contrast to HR, non-homologous end-joining (NHEJ) is a process by which the broken DNA ends are joined directly without the aid of an intact template. The classical NHEJ involves DNA end-binding by the Ku70/Ku80 heterodimer that bridges DNA ends and protects them from extensive resection. Ku forms a toroidal structure that binds DNA by sliding a DSB end through its opening and functions as a docking site for NHEJ repair factors [4-6]. In Saccharomyces cerevisiae, DSB ends are processed by MRX, consisting of Mre11p, Rad50p, and Xrs2p, and DNA polymerase Pol4 to create compatible, ligatable ends. After processing, ends are ligated by the dedicated NHEJ ligase IV, composed of DNA ligase 4 (Dnl4p), Lif1p, and Nej1p $[7,8]$. Overhang polarity makes a significant difference in terms of factor recruitment and repair efficiency, with DSBs containing $5^{\prime}$-overhangs repaired more efficiently than DSBs with $3^{\prime}$ overhangs, but with a greater probability of induced mutation [9].

The choice between HR and NHEJ is in large part determined by the extent of DNA end resection, as extensive resection is inhibitory to NHEJ and channels the lesion towards the HR repair path- 
way. In yeast, end resection is initiated by the combined action of the endonuclease Sae2p and the MRX complex, followed by more extensive resection by the helicase-nuclease complex that contains Sgs1p, Top3p, Rmi1p, and Dna2p and by the exonuclease Exo1p. The endonuclease activity of Mre11p, which is promoted by Sae2p, nicks the $5^{\prime}$-terminated DNA strand in the vicinity of the DSB end, following which its $3^{\prime}$ to $5^{\prime}$ exonuclease activity degrades the DNA towards the DSB end. This generates a 3'-tailed substrate for Sgs1p/Dna2p and/or Exo1p, which degrade the DNA in the opposite direction to generate more extensive resection [10-12]. Ku prevents the Exo1p- and Sgs1p/Dna2p-dependent resection [13].

An alternative end-joining (A-EJ) pathway has been described and proposed to function as a "plan B", both for classical NHEJ and for HR, as it operates on DNA ends that cannot be processed by the initially chosen repair pathway. This end joining activity is independent of $\mathrm{Ku}$ and ligase IV and is unmasked in absence of these factors [14]. This pathway, which is poorly characterized and may be comprised of multiple distinct mechanisms, is also referred to as backup NHEJ or microhomology-mediated end-joining; it is enhanced by microhomologies, suggesting that A-EJ is initiated by DNA end resection, an inference supported by the involvement of Sae2p (the equivalent of mammalian CtIP) and Mre11p [15-18]. Since Ku inhibits extensive end resection, it also inhibits A-EJ. A-EJ is a highly error-prone process and often associated with deletions at the repair junctions, and it may contribute to genomic instability such as chromosomal translocations [15,19].

We have previously investigated the role of the yeast high mobility group (HMGB) protein Hmo1p (Fig. 1A) in repair of a genomic DSB. Absence of Hmo1p results in more efficient repair by both HR and NHEJ, an effect that was attributed to a change in chromatin remodeling events that precede repair, including faster eviction of nucleosomes in the vicinity of the DSB combined with faster DNA end resection [20-22]. The absence of Hmo1p also leads to increased sensitivity of chromatin to digestion by micrococcal nuclease, a sensitivity that is fully reversed when mammalian histone $\mathrm{H} 1$ is expressed in hmo1 $\Delta$ cells. These observations led to the inference that Hmo1p has linker histone-like functions; it stabilizes chromatin and it must be evicted along with core nucleosomes to enable DSB repair. Hmo1p occupancy across the yeast genome is variable, ranging from below background levels to particular enrichment at sites such as rDNA and ribosomal protein gene promoters [23]. In addition to its ability to stabilize conventional nucleosome arrays, Hmo1p has also been reported to stabilize nucleosome-free DNA and DNA regions characterized by low nucleosome occupancy $[22,24]$.

The reported role of Hmo1p in controlling resection raises the possibility of its direct participation in repair pathway choice. Using a plasmid end-joining assay as a read-out for end-joining to separate roles of Hmo1p in the repair process from its effects on chromatin structure, we report here that transformation of an $h m o 1 \Delta$ strain with linear plasmid DNA with cohesive ends results in significantly reduced transformation efficiency, suggesting that Hmo1p promotes DNA end-joining. We propose that Hmo1p controls DNA resection, thereby favoring the more efficient classical NHEJ over A-EJ. In absence of Ku, HMO1 deletion reduces repair efficiency further, suggesting that excessive DNA resection is inhibitory for A-EJ.

\section{Materials and methods}

\subsection{Strain construction}

$D D Y 3$ is isogenic to $W 303-1 A$. The $D D Y 1299$ derivative of $D D Y 3$ in which $H M O 1$ is deleted, strain $H M O 1-A B$, which encodes a truncated version of Hmo1p deleted for its lysine-rich C-terminal extension, and strain expressing Hmo1 p-FLAG were previously described [25]. The gene encoding Ku80p was deleted by amplifying the URA3 marker with primers that include $\sim 80 \mathrm{nt}$ of flanking sequence homologous to the KU80 gene, followed by transformation of either DDY3 or DDY3hmo1 $\Delta$ haploid cells to generate $k u \Delta$ and ku $\Delta h m o 1 \Delta$, respectively.

\subsection{Yeast high efficiency transformation}

Cells were grown in YPD (1\% yeast extract, $2 \%$ peptone, $2 \%$ glucose) at $30^{\circ} \mathrm{C}$ with constant shaking to an optical density at $600 \mathrm{~nm}$ of 0.8 , and the pelleted cells were washed with $1 \mathrm{X}$ phosphatebuffered saline (PBS) and resuspended in 1X Tris, EDTA, and lithium acetate buffer (TEL) and were left on a nutator overnight at room temperature. The next day cells were pelleted and resuspended in $100 \mu \mathrm{l} 1 \mathrm{X}$ TEL per $10 \mathrm{ml}$ culture and incubated at room temperature for $30 \mathrm{~min}$. One hundred microliter of competent cells, $10 \mu \mathrm{l}$ of carrier DNA (sheared salmon sperm DNA) and $1 \mu \mathrm{g}$ of plasmid DNA was mixed in a microcentrifuge tube and incubated for $30 \mathrm{~min}$. Seven hundred microliter of $40 \%$ polyethylene glycol (PEG) in $1 \mathrm{X}$ TEL was added to each tube, mixed well, and incubated at room temperature for $60 \mathrm{~min}$ without shaking. Eightyeight microliter of dimethyl sulfoxide (DMSO) was added to each tube, mixed, and the cells were subjected to heat shock at $42^{\circ} \mathrm{C}$ for $45 \mathrm{~min}$. The cells were spun gently at $8000 \mathrm{rpm}$ for $30 \mathrm{~s}$, pellets were washed with $300 \mu \mathrm{l}$ water, and cells were resuspended in $400 \mu$ l water. Two hundred microliter was plated on SD drop out media lacking leucine; where indicated, $0.5 \mathrm{mM}$ caffeine was included in the media.

\subsection{Plasmid end-joining assay}

Plasmid pMV1328 (LEU marker) [26] was linearized by digestion with Ncol (to generate cohesive ends with a four-nucleotide 5'-overhang), with AsiSI (to generate cohesive ends with a twonucleotide $3^{\prime}$-overhang), or with Nrul (to generate blunt ends); all enzymes cut within the KanMX6 coding sequence (that has no homology with the yeast genome). Efficiency of digestion was verified by agarose gel electrophoresis and DNA was purified by phenol-chloroform extraction and ethanol-precipitation. To verify that undigested DNA did not contribute significantly to the measured repair accuracy, pMV1328 was also double-digested with the blunt-cutters Ncol and SmaI to remove a segment of the KanMX6 gene such that ligation of the blunt-ended plasmid DNA cannot restore resistance to G418. Both linearized and circular plasmids were used to transform DDY3 (wild-type) and mutant cells. After 3-4 days incubation, at least 50 colonies were counted for each transformation. Plasmid repair efficiency was calculated as the ratio of the number of stable transformants obtained when cells were transformed with linearized versus circular plasmid DNA. Data were also normalized to the repair efficiency of wild type DDY3. To calculate repair accuracy, $\mathrm{Leu}^{+}$transformants were replica-plated on YPD agar medium containing $0.3 \mathrm{mg} / \mathrm{ml} \mathrm{G} 418$ to select for cells expressing an intact KanMX6 gene. To verify repair efficiency, plasmids pRS416 (URA3 marker) and pRS414 (TRP marker) were linearized with HindIII (to generate a four-nucleotide $5^{\prime}$-overhang) and SacI (to generate a four-nucleotide $3^{\prime}$-overhang), respectively; both enzymes cut within the vector backbone that has no homology within the yeast genome. Yeast cells were transformed as described above and plated on drop-out media selecting for the appropriate marker.

All experiments were independently performed at least three times. Error bars represent standard deviation. Two tailed student $t$-test was used to calculate the P values. 
A

Box A

Box B

K-rich

Hmo1p

B

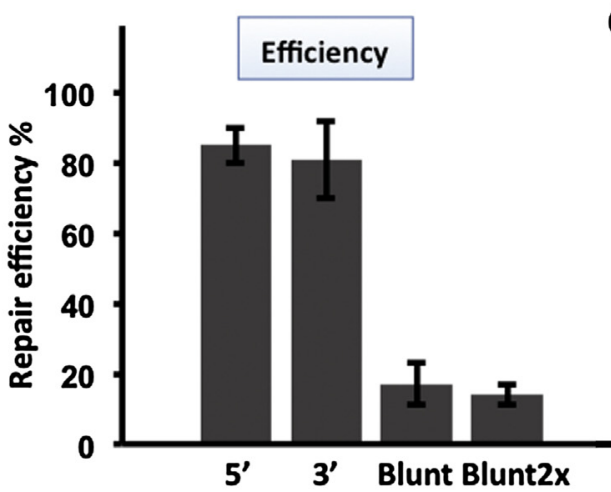

D

5'-overhang

$\mathbf{F}$
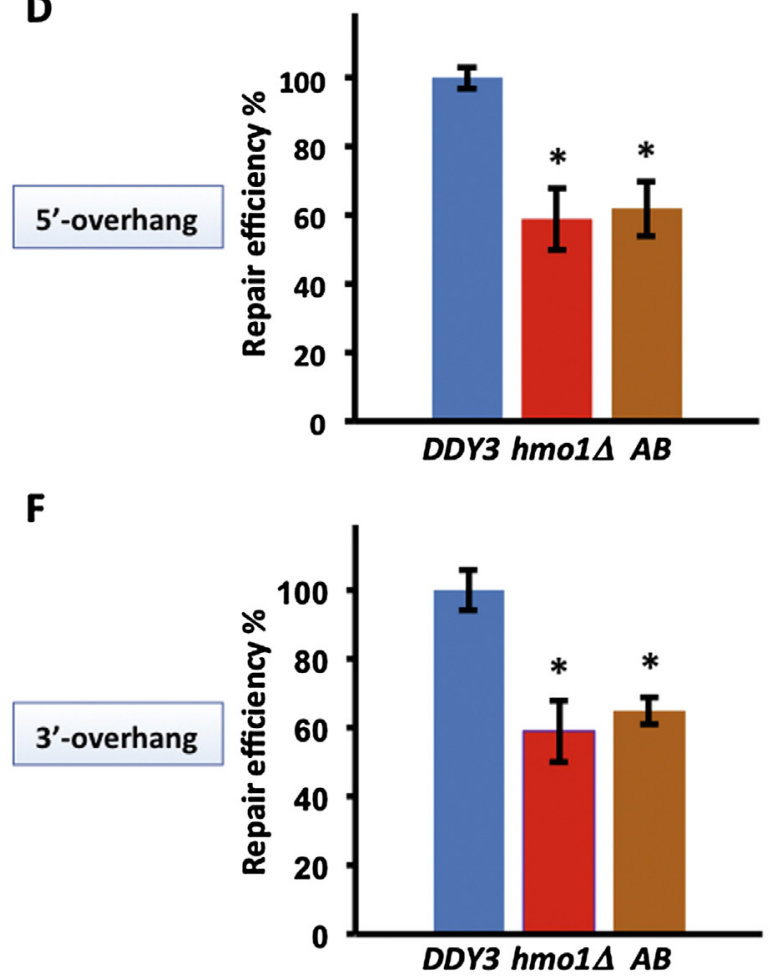

H

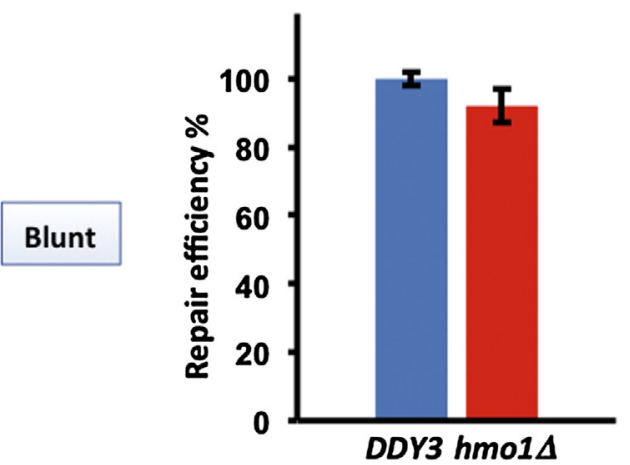

C

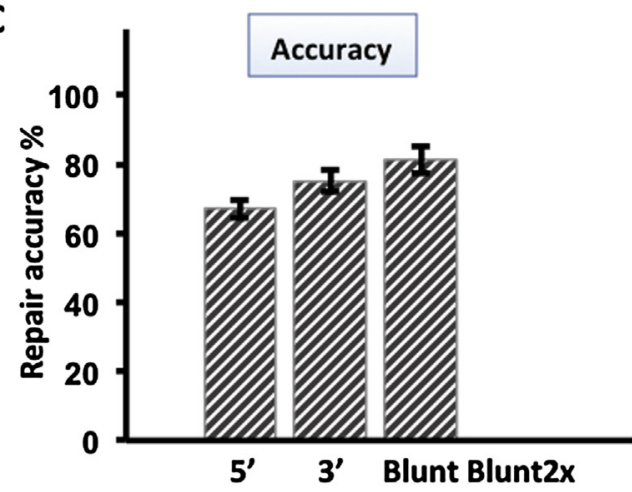

$\mathbf{E}$

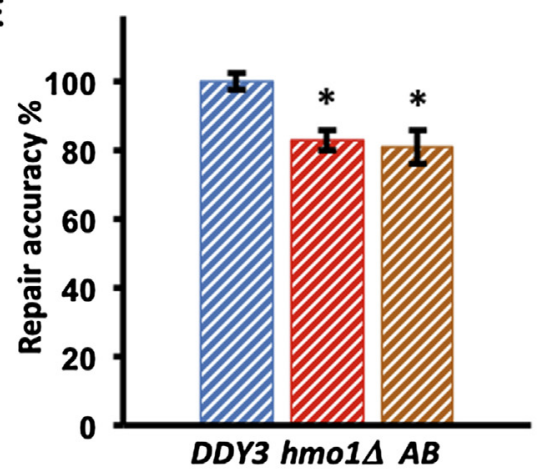

$\mathbf{G}$

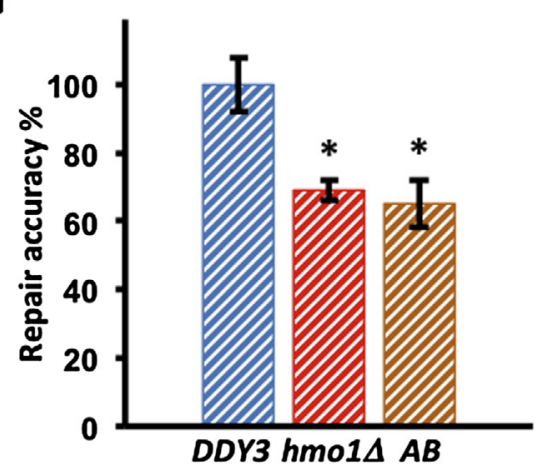

I

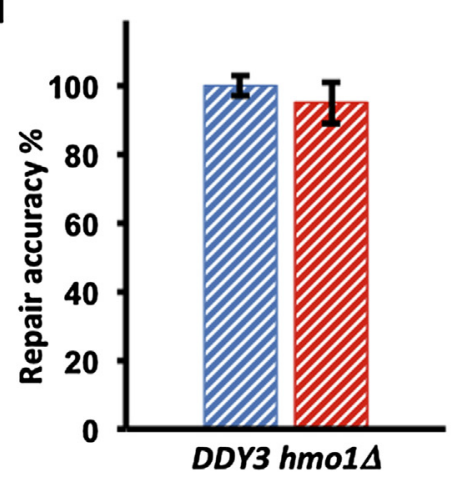

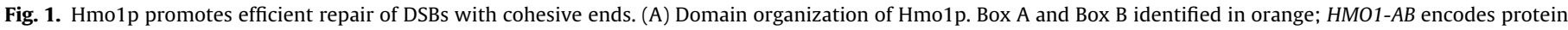

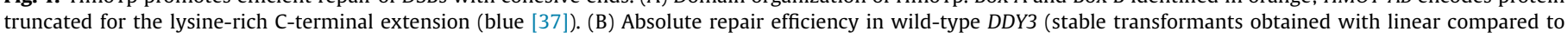

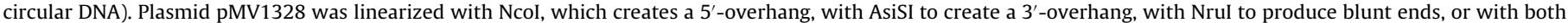

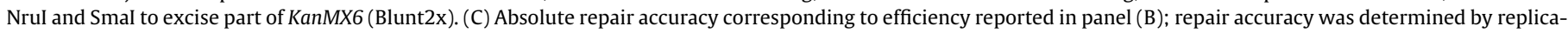

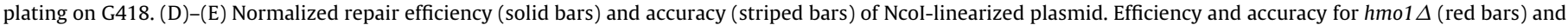

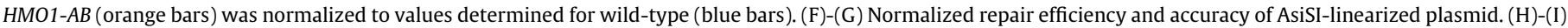

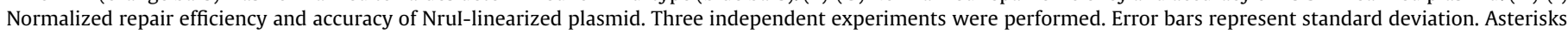
indicate a significant difference from wild type $(\mathrm{P}<0.05)$. 


\subsection{DNA end resection}

To induce genomic DSB, the galactose-inducible $\mathrm{HO}$ endonuclease gene was furnished on a centromeric plasmid (URA3 or TRP marker) and transformed in $D D Y 3$ or mutant cells. Cells were grown in $\mathrm{SD}$ drop out media at $30^{\circ} \mathrm{C}$ to an $\mathrm{OD}_{600}$ of 1.0 ; where indicated, caffeine was included at a final concentration of $0.5 \mathrm{mM}$. DSB was induced by addition of $2 \%$ galactose. Cells were harvested after DSB induction times of $20 \mathrm{~min}, 1 \mathrm{~h}, 2 \mathrm{~h}, 3 \mathrm{~h}$ and $4 \mathrm{~h}$. Genomic DNA was extracted by vortexing cells with glass beads and phenol. Twenty microliters of genomic DNA sample (60 ng in 1X NEB Exonuclease I buffer) was digested with 20 units of $E$. coli exonuclease I at $37^{\circ} \mathrm{C}$ overnight. The level of DNA resection adjacent to the specific DSB was measured by qPCR using primers annealing $1.6 \mathrm{~kb}$ or $3.1 \mathrm{~kb}$ upstream of the DSB [20]. All cycle threshold $\left(C_{t}\right)$ values were normalized to values for an independent locus on chromosome 5 (POL5); a higher $\mathrm{C}_{\mathrm{t}}$ reflects a less abundant template. The assay was repeated three times and average and standard deviations (SD) are reported.

\subsection{Gene expression}

Expression of genes encoding DNA repair or DNA damage response factors Sae2p, Dna2p, and Rnr3p was analyzed using qRTPCR using primer pairs Sae2F 5'-AGCAGAGGTTCACTATCGTAATG-3' and Sae2R 5'-CAGAGCAATCTTCCAAATCGC-3', Dna2F 5'-TGAGAAACGCAAGAGTGAGAG-3' and Dna2R 5'-CCTGTTTTGGCTTGATTGTCTAC-3', and RNR3F 5'-CAAAGAGCCCGTTCAATTC-3' and RNR3R 5'TAACACCGGAGTACACACCAG-3' ${ }^{\prime}$, respectively. Cells were grown at $30{ }^{\circ} \mathrm{C}$ to an $\mathrm{OD}_{600}$ of $\sim 0.8$; where indicated, caffeine was added at a final concentration of $0.5 \mathrm{mM}$. One $\mathrm{ml}$ culture was removed to extract RNA; cells were mixed with ice-cold diethylpyrocarbonate (DEPC)-treated water, centrifuged, and the pellet was frozen at $-80^{\circ} \mathrm{C}$. Total RNA was isolated using the illustra RNAspin Mini Isolation kit (GE Healthcare) and DNA contamination was removed using Turbo DNase (Ambion); absence of DNA was verified by PCR and RNA was quantified using NanoDrop. The cDNA was prepared using $500 \mathrm{ng}$ total RNA in $1 \mathrm{X}$ AMV reverse transcriptase buffer with $1 \mathrm{mM} \mathrm{MgCl} 2,1 \mathrm{mM}$ dNTP, and 10 units of AMV reverse transcriptase (New England BioLabs) in a total reaction volume of $25 \mu \mathrm{l}$. The mixture was incubated at $42^{\circ} \mathrm{C}$ for $1 \mathrm{~h}$. A ViiA ${ }^{\mathrm{TM}} 7$ (Applied Biosystems) was used for qPCR using Taq polymerase (New England BioLabs) for amplification and SYBR Green I (Sigma) for detection. For analysis, IPP1 (encoding inorganic pyrophosphatase) was used as a reference gene, and expression of target genes was normalized to expression levels measured in each strain prior to induction of DSB. Each experiment was repeated three times and average and standard deviations (SD) are reported.

\section{Results}

\subsection{Absence of Hmo1p results in reduced repair efficiency and accuracy}

To separate the roles of Hmo1p in chromatin remodeling from its roles in NHEJ, we used the plasmid end-joining assay. A DSB was introduced in plasmid pMV1328 using Ncol, which cuts the KanMX6 coding sequence to create a 5'-overhang, AsiSI, which generates a 3'-overhang, or NruI, which produces a blunt cut [26]. Since the KanMX6 sequence has no homologous sequence within the yeast genome with which to initiate repair by HR, the DSB in the plasmid DNA must be repaired by NHEJ to allow cells to survive in the selection media. Repair efficiency was quantified as the number of $\mathrm{Leu}^{+}$transformants relative to transformation with supercoiled plasmid DNA (Fig. 1B). While repair efficiency of DNA with $3^{\prime}$ - or $5^{\prime}$-overhangs was similar ( $\left.80-85 \%\right)$, the assay reflected the expected low efficiency of repair of DNA with blunt ends. To quantify the proportion of mutagenic ligation events, $\mathrm{Leu}^{+}$transformants obtained on repair of linearized pMV1328 were replica-plated on G418 plates. Since the cuts were introduced in the coding region of KanMX6, a functional gene and hence G418 resistance will be inherited only if the end-joining is accurate. For all DNA repair events, accuracy was $\sim 70-80 \%$ (Fig. 1C). When pMV1328 was double-digested with two blunt-cutters that remove a segment of KanMX6, repair efficiency was similar to that obtained for repair of DNA with a single cut (14\%), whereas no G418-resistant colonies were observed (Blunt2x).

Compared to wild-type $D D Y 3$, the hmo1 $\Delta$ mutant exhibited significantly reduced repair efficiency for repair of DNA with either $5^{\prime}$ or 3'-overhangs (Fig. 1D,F; showing repair efficiency normalized to that observed in DDY3). By contrast, there was no significant reduction in repair efficiency in cells deleted for HMO1 for repair of a blunt-ended DSB (Fig. $1 \mathrm{H})$. Similarly, transformation of $h m o 1 \Delta$ with plasmids pRS416 or pRS414 linearized with HindIII (to generate a four-nucleotide 5'-overhang) and Sacl (to generate a four-nucleotide 3 '-overhang), respectively, yielded a significantly reduced repair efficiency compared to wild-type (data not shown). These data suggest that Hmo1p promotes efficient NHEJ, but only for repair of DNA with protruding ends. The hmo1 $\Delta$ strain also repaired DSBs with cohesive ends less accurately compared to wild-type, whereas accuracy of repair of blunt-ended DNA was unaffected by deletion of Hmo1p (Fig. 1E,G,I).

\subsection{Requirement of the Hmo1p lysine-rich C-terminal tail for efficient NHEJ}

Hmo1p has two DNA binding domains, box $\mathrm{A}$ and box $\mathrm{B}$, and a lysine-rich C-terminal tail (Fig. 1A). This C-terminal extension is indispensable for DNA bending and for stabilizing chromatin structure, and it is required for bridging of DNA ends [20,27,28]. To test if the Hmo1p C-terminal tail is required for efficient NHEJ, we used strain $H M O 1-A B$, which encodes a truncated version of $H m o 1 p$ deleted for its lysine-rich C-terminal extension. Repair of DSBs in $H M 01-A B$ mimicked the repair phenotype characteristic of hmo1 $\Delta$, with cohesive end repair being less efficient in HMO1-AB than in wild-type cells (Fig. 1D,F). Similarly, repair accuracy was lower in $H M O 1-A B$ compared to wild-type for repair of DNA with protruding ends (Fig. 1E,G). This suggests that the C-terminal domain of Hmo1p is required for efficient NHEJ and for fidelity.

\subsection{Ku and Hmo1p function in different repair pathways}

Ku participates in classical NHEJ repair of DNA with overhangs whereas repair of DNA with blunt ends does not depend on $\mathrm{Ku}$ $[26,29,30]$. Consistent with expectations, we found that deletion of Ku significantly reduced NHEJ repair of DNA with 5'-overhangs, but not of DNA with blunt ends (Fig. 2A,C). Repair in the absence of Ku of DNA with protruding ends is a result of error prone A-EJ, and we observed the expected low accuracy of repair in $k u \Delta$ cells (Fig. 2B). In a ku80 $\Delta$ background, inactivation of HMO1 resulted in a further reduction in efficiency of repair of DNA with cohesive ends (Fig. 2A). Notably, this suggests that Ku and Hmo1p function in separate repair pathways. Repair accuracy in $k u \Delta h m o 1 \Delta$ cells was extremely low, with very few colonies observed upon replica-plating on G418. By contrast, repair efficiency and accuracy in $k u \Delta h m o 1 \Delta$ and $k u \Delta$ cells was similar to wild-type for DNA with blunt ends (Fig. 2C-D). 

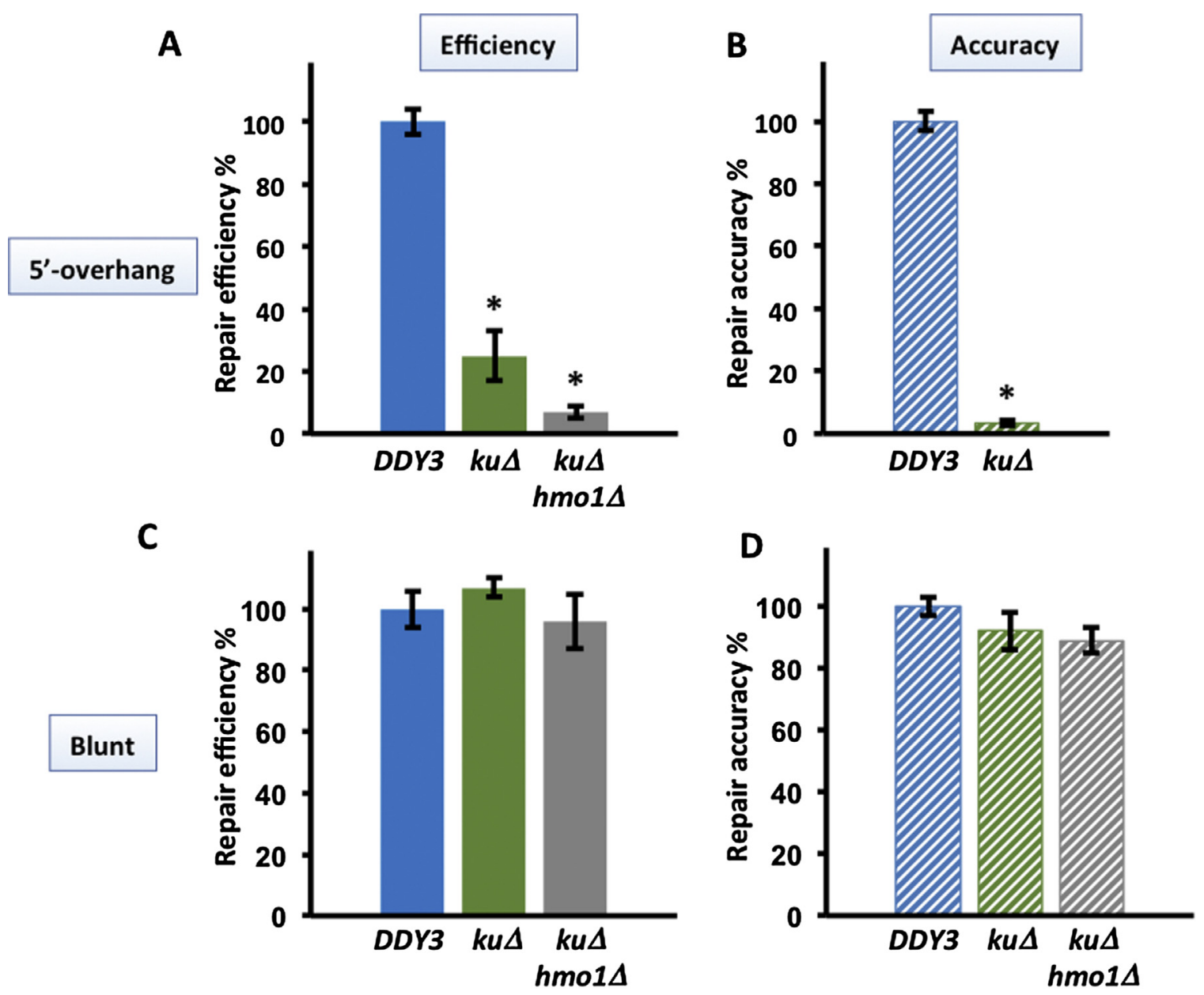

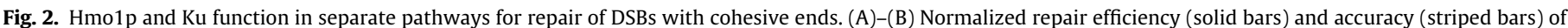

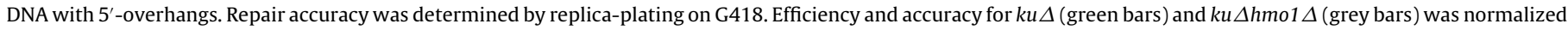

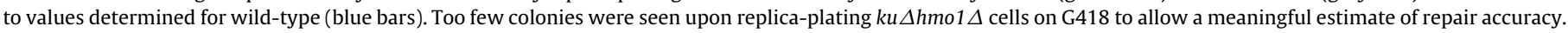

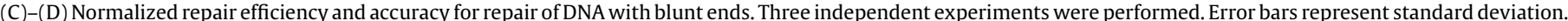
Asterisks indicate a significant difference from wild type $(\mathrm{P}<0.05)$.

\subsection{Hmo1p controls caffeine-sensitive resection}

Caffeine has been reported to reduce DNA end resection by inducing degradation of Sae2p and rapid turnover of Dna2p [31]. Since the absence of $\mathrm{Hmo1}$ p results in increased DNA resection at a chromosomal DSB in vivo [20], we wondered if excessive resection might be the cause of reduced NHEJ repair efficiency in hmo1 $\Delta$. To address this, we performed the plasmid end joining assay by using NcoI to generate a DSB with 5'-overhangs in pMV1328 and selected transformants on agar plates lacking leucine and containing $0.5 \mathrm{mM}$ caffeine; this caffeine concentration was previously shown to inhibit resection, yet it is much lower than the concentration required to inhibit checkpoint kinases [31]. In the presence of caffeine, the reduced repair efficiency of $h m 01 \Delta$ was restored to the level observed in wild-type cells (Fig. 3A; compare solid and striped red bars). Similarly, caffeine restored the repair efficiency of $k u \Delta h m o 1 \Delta$ cells to the levels observed in $k u \Delta$. In wild-type $D D Y 3$, caffeine also increased relative repair efficiency (resulting in $\sim 100 \%$ absolute efficiency compared to the efficiency for DDY3 not plated with caffeine (Fig. 1B); compare solid and striped blue bars). No effect of caffeine was observed when cells repaired blunt ended DSBs, whether or not cells were deleted for KU80 and HMO1 (Fig. 3B). Evidently, addition of caffeine enhances repair of DNA with 5'-overhangs, but not of DNA with blunt ends.
To verify that caffeine can reverse the increased DNA resection phenotype of hmo1 $\Delta$, we analyzed DNA end resection following induction of a DSB in vivo in the presence of caffeine. DSB was induced by $\mathrm{HO}$ endonuclease, which generates 3 '-overhangs. Following incubation of genomic DNA with exonuclease to digest the single-stranded overhangs produced after DNA resection, qPCR was performed to measure levels of residual (i.e., not resected) DNA template and the $C_{t}$ values were normalized to that for the reference site (POL5); in this assay, higher levels of resection are associated with less abundant template and therefore a greater $C_{t}$ value. The gradual disappearance of template was also verified by agarose gel electrophoresis; for example, resection was visually detected $1.6 \mathrm{~kb}$ upstream of the DSB in wild-type DDY3 3-4 h after DSB induction, with faster loss of template observed in $h m o 1 \Delta$, reflecting more efficient resection (Fig. 3D). Quantitation of residual template verified the loss of template after DSB induction in DDY3 (Fig. 3C). Deletion of either HMO1 or KU80 resulted in more efficient resection compared to wild-type cells, and simultaneous deletion of both HMO1 and KU80 yielded modestly greater levels of resection compared to deletion of either gene alone. When measuring resection further from the break site ( $3.1 \mathrm{~kb}$ upstream), a similar pattern was observed with lower levels of resection in wildtype and in $k u \Delta$ and $h m o 1 \Delta$ strains compared to closer to the break site, whereas resection in $k u \Delta h m o 1 \Delta$ cells was significantly more efficient com- 
A

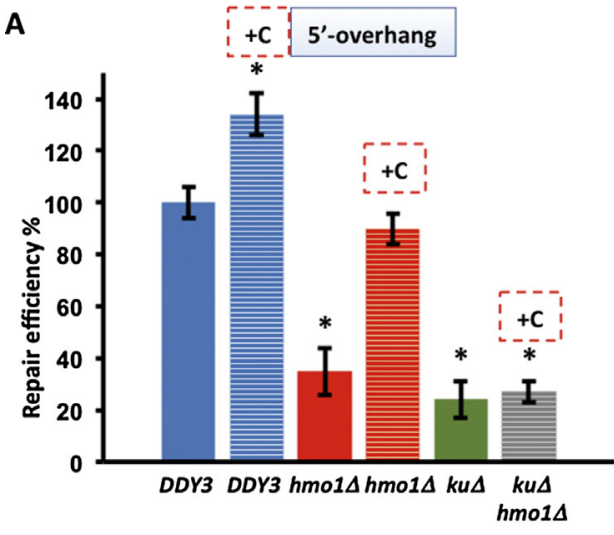

C

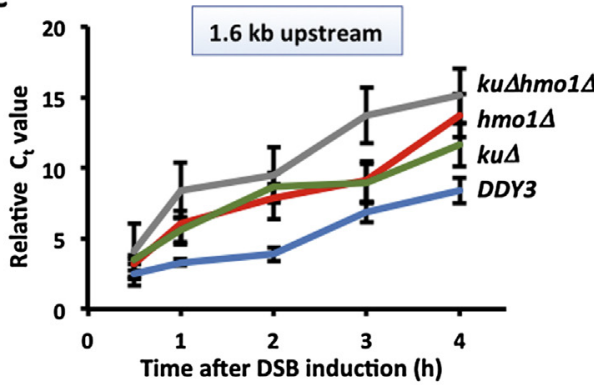

E

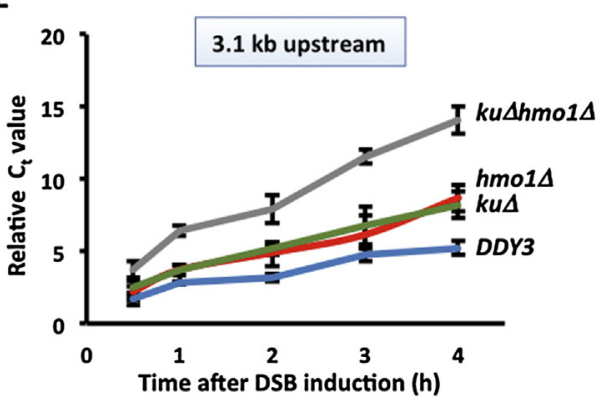

B

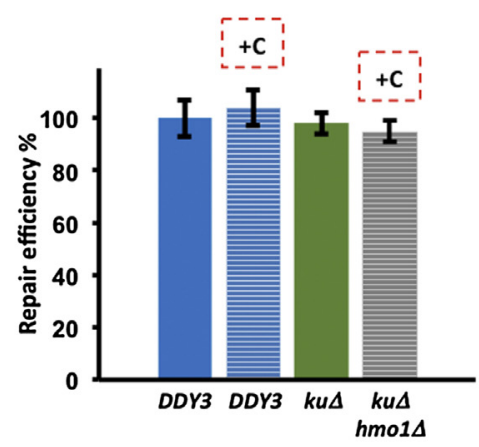

D

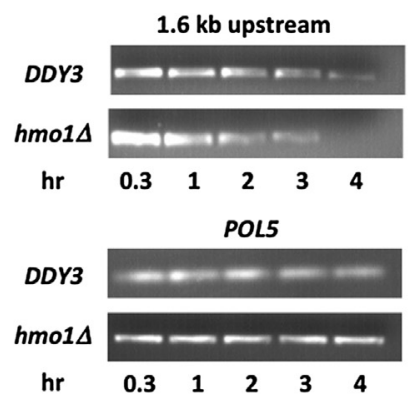

$\mathbf{F}$

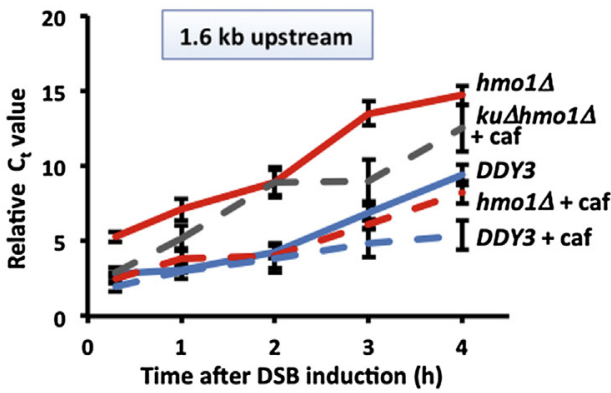

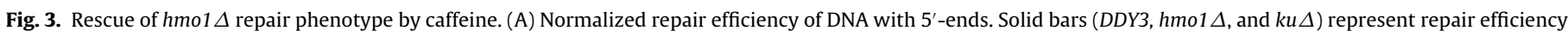

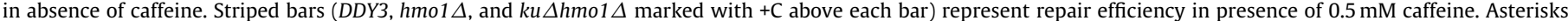

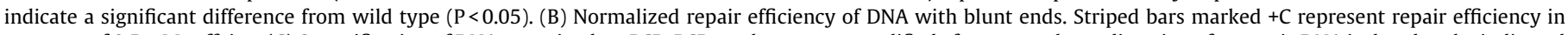

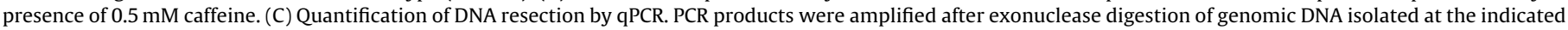

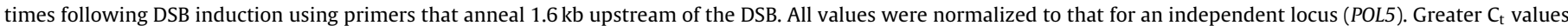

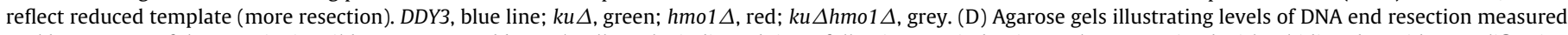

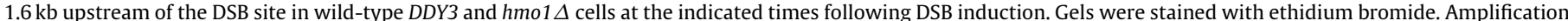

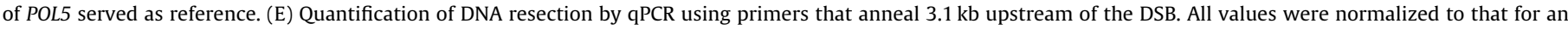

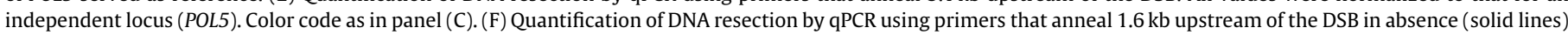
or presence of $0.5 \mathrm{mM}$ caffeine (dashed lines). Color code as in panel (C). Three independent experiments were performed. Error bars represent standard deviation.

pared to cells deleted for a single gene (Fig. 3E). This indicates that Hmo1p and Ku function to limit DNA end resection with comparable efficiency and that simultaneous deletion of both proteins results in an additive effect and even more extensive resection.

In the presence of caffeine, the kinetics of DNA resection were reduced. In wild-type cells, caffeine caused significantly reduced resection after prolonged DSB induction ( $4 \mathrm{~h}$; Fig. $3 \mathrm{~F}$; compare solid and dashed blue lines). By comparison, caffeine reduced resection in $h m o 1 \Delta$ to the level observed in wild-type cells, even when measured shortly after DSB induction (Fig. 3F; compare solid and dashed red lines). While caffeine also reduced resection in $k u \Delta h m o 1 \Delta$ cells, the level of resection was still greater than that observed in wild-type cells (dashed grey line). This suggests that Hmo1p reduces an aspect of DNA end resection that is sensitive to caffeine; in contrast, the elevated levels of DNA resection in $k u \Delta h m o 1 \Delta$ cells was not fully restored to wild-type levels on addition of caffeine, likely reflecting that absence of Ku facilitates a caffeine-insensitive DNA resection process.

The initiation of resection requires the MRX complex and Sae2p, whereas more extensive resection depends on Exo1 and the concerted action of Dna2p and Sgs1p; both Sae2p and Dna2p accumulate after induction of a DSB, and this accumulation is not observed in presence of caffeine [31]. To determine if the effect of Hmo1p on DNA end resection was due to altered expression of either SAE2 or DNA2, we performed qRT-PCR before and after DSB induction. No difference in expression of either gene was observed in hmo1 $\Delta$ cells (Fig. 4A-B).

The cellular response to DNA damage includes a block of progression through the cell cycle to allow sufficient time for repair and induction of genes involved in DNA repair. One of the genes that are induced by DNA damage is RNR3, which encodes a large subunit of ribonucleotide reductase (RNR) that catalyzes the rate- 
A

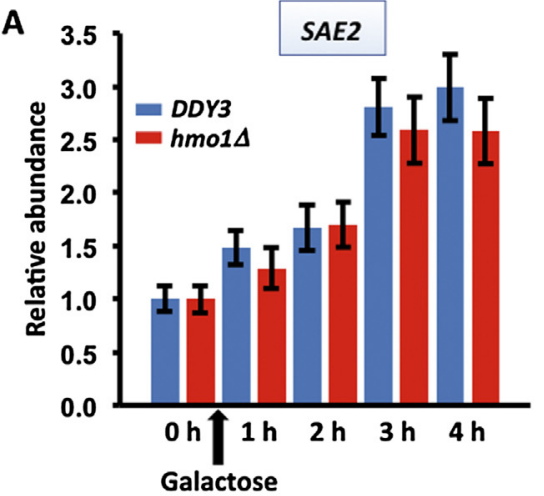

C

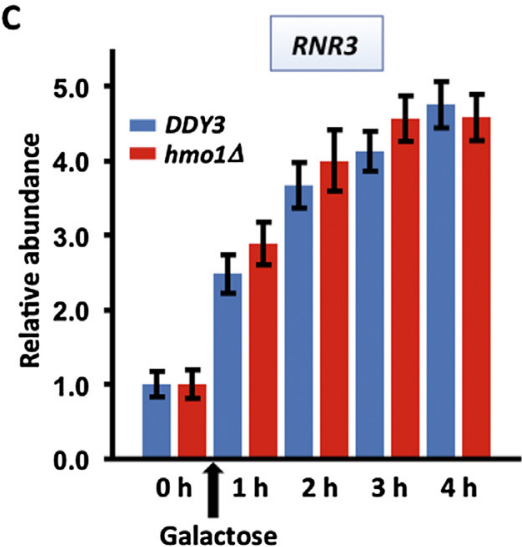

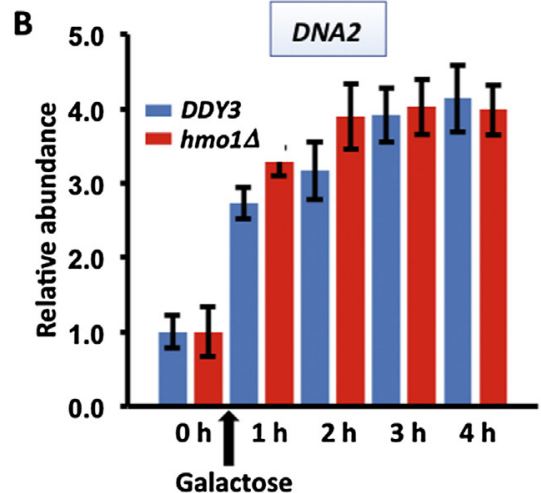

D

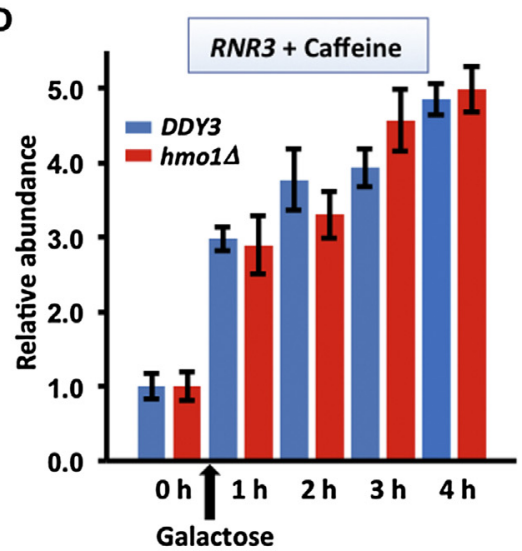

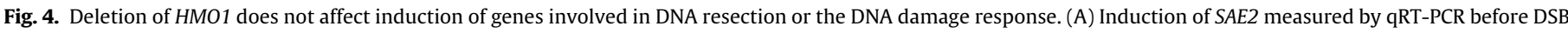

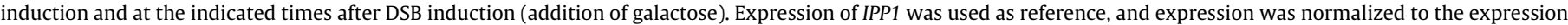

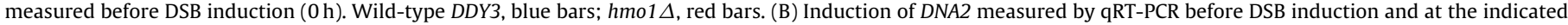

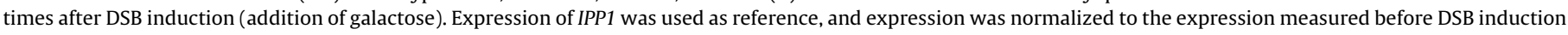

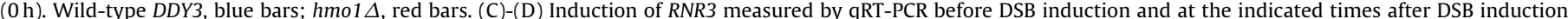

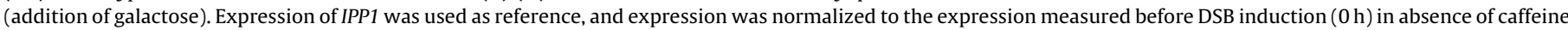

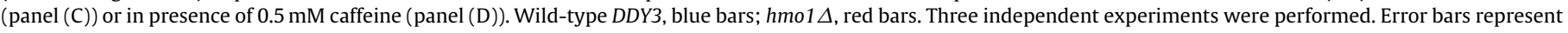
standard deviation.

limiting step in synthesis of dNTPs [32,33]. A series of events that includes recruitment of the checkpoint kinases Mec1p and Tel1p to the DSB propagate the DNA damage signal, ultimately resulting in events such as prevention of mitosis and upregulation of $R N R 3$ [34]. It was previously reported that caffeine inhibits DNA end resection, independent of its inhibition of checkpoint kinases [31]. To verify that concentrations of caffeine used for inhibition of resection do not interfere with checkpoint signaling, we measured induction of $R N R 3$ in absence and presence of $0.5 \mathrm{mM}$ caffeine. No significant attenuation of $R N R 3$ induction was observed in presence of this concentration of caffeine, nor was RNR3 induction affected by deletion of HMO1 (Fig. 4C-D).

\section{Discussion}

\subsection{Hmo1p promotes end-joining of DSBs with cohesive ends}

The direct sealing of broken DNA ends is primarily achieved by classical NHEJ. However, in absence of critical NHEJ components such as Ku, cells resort to A-EJ, which is initiated by DNA end resection to use micro-homologies of $<15$ nucleotides $[15,19]$. In the context of a nucleosomal array, Hmo1p stabilizes chromatin, which slows chromatin remodeling, perhaps to protect the compromised DNA and ensure optimal timing of repair factor recruitment to the break site. The data reported here suggest that Hmo1p, which in many contexts associates with nucleosome-free DNA, in addition promotes end-joining of DNA with cohesive ends. Since deletion of HMO1 leads to reduced repair efficiency and accuracy in presence of Ku (Fig. 1D-E), and since classical NHEJ is predominant in presence of $\mathrm{Ku}$, our data suggest that absence of Hmo1p directs a significant proportion of repair events to less efficient and more error-prone A-EJ pathways. Since both repair efficiency and accuracy of $k u \Delta h m o 1 \Delta$ cells is much lower than that observed on deletion of either gene alone (Figs. Fig. 11D-E and Fig.22A-B), we also infer that absence of Hmo1p compromises the A-EJ repair that would be the repair pathway of choice when classical NHEJ is not an option.

DSBs increase the occurrence of chromosomal translocations if they are repaired by microhomology-dependent A-EJ and microhomologies are present distant from the break site [19]. DSBs therefore have high mutagenic potential. Even a two-fold reduction in repair efficiency as observed in absence of Hmo1p (Fig. 1) is therefore likely to be physiologically relevant, particularly as it is associated with reduced accuracy, which we infer to reflect a contribution from A-EJ to the overall repair efficiency.

\subsection{Hmo1p controls an aspect of DNA end resection that is sensitive to caffeine}

Our data suggest that the mechanism by which Hmo1p suppresses A-EJ repair of DNA with cohesive ends is by controlling DNA end resection. The first step in resection is executed by MRX and Sae2p, which create a short 3'-tailed substrate for more extensive $5^{\prime}$ to $3^{\prime}$ resection by two parallel pathways that depend on 
Exo1p or the helicase/nuclease complex that contains Sgs1p and Dna2p. MRX-Sae2p has also been implicated in recruiting both Exo1p and Sgs1p/Dna2p and in stimulating their activity $[7,10,13]$. While Ku-binding to DSB ends inhibits the Exo1- and Sgs1p/Dna2pdependent processing in absence of MRX, absence of Ku bypasses the requirement for initiation of resection by MRX and permits direct processing by Exo1p and Sgs1p/Dna2p [13]. Loss of endprotection by $\mathrm{Ku}$ therefore results in excessive end-processing, even in absence of MRX. Consistent with this expectation, we observe increased resection in $k u \Delta$ cells (Fig. 3C,D).

At a genomic DSB, absence of Hmo1p also results in increased DNA end resection, whereas deletion of both $\mathrm{Ku}$ and $\mathrm{Hmo} 1 \mathrm{p}$ is additive and leads to a further increase in resection beyond that seen on deletion of either protein alone (Fig. 3C-F). The hmo1 $\Delta$ repair phenotype is rescued by caffeine; in contrast, addition of caffeine only attenuates resection in $k u \Delta h m o 1 \Delta$ cells, but does not completely restore levels of resection to that observed in wild-type cells (Fig. 3F). Caffeine promotes the proteasomal degradation of Sae2p and Dna2p, but does not change levels of Exo1p [31]. Since Ku interferes with resection by Exo1p and Sgs1p/Dna2p [13,35], an interpretation that is consistent with the observation that resection remains elevated in caffeine-treated $k u \Delta h m o 1 \Delta$ cells is therefore that Exo1p-mediated processing is primarily responsible for the increased resection. By extension, is it conceivable that Hmo1p interferes only with processes that require the caffeine-sensitive Sae2p and/or Dna2p. Since MRX-Sae2p initiates resection, we favor the interpretation that Hmo1p suppresses this step, since initiation of resection by MRX-Sae2p should allow further resection by both Sgs1p/Dna2p and the caffeine-insensitive Exo1p. Regardless of specific target, our data show that Hmo1p suppresses DNA end resection; a functional consequence of this would be that repairs are directed towards the more accurate NHEJ. This interpretation is supported by the observation that repair efficiency and accuracy is compromised in hmo1 $\Delta$ cells (Fig. 1 ). We propose that excessive resection in $h m o 1 \Delta$ interferes with classical NHEJ by producing a tailed substrate (created by MRX/Sae2p) with lower affinity for $\mathrm{Ku}$ but higher affinity for Exo1p and/or Sgs1p/Dna2p, reducing classical NHEJ repair efficiency and forcing cells to repair by A-EJ. This interpretation is consistent with reports that deletion of SAE2 promotes NHEJ [36].

We also note that repair efficiency is lower in $k u \Delta h m o 1 \Delta$ than in $k u \Delta$ for repair of DSBs with $5^{\prime}$-overhangs. Since $k u \Delta$ cells repair DSBs by A-EJ, an implication of this observation is that Hmo1p functions in A-EJ, most likely by ensuring optimal levels of resection. Since caffeine restores repair efficiency to levels observed in $k u \Delta$, our data also suggest that the extensive resection that is characteristic of the ku $\Delta h m o 1 \Delta$ double mutant (Fig. 3) is inhibitory to A-EJ.

Neither Ku, nor Hmo1p are required for repair of DSBs with blunt ends. We also saw no effect of caffeine on the efficiency of DNA repair, which suggests that Sae2p/Dna2p are not involved. Since deletion of HMO1 or addition of caffeine had no effect on efficiency of repair of blunt-ended substrates, our interpretation is that DNA resection (at least the component that is controlled by Hmo1p and inhibited by caffeine) is not required. Thus, we infer that Hmo1p suppresses A-EJ repair of DNA with protruding ends only. Indeed, A-EJ is likely comprised of multiple mechanisms that may for example depend on the length and position of microhomologies [19].

\section{Conflict of interest}

The authors declare that there are no conflicts of interest.

\section{Funding}

This research did not receive any specific grant from funding agencies in the public, commercial, or not-for-profit sectors.

\section{Acknowledgements}

We thank Michael Volkert for the pMV1328 plasmid.

\section{References}

[1] E. Mladenov, S. Magin, A. Soni, G. Iliakis, DNA double-strand-break repair in higher eukaryotes and its role in genomic instability and cancer: cell cycle and proliferation-dependent regulation, Semin. Cancer Biol. 37-38 (2016) 51-64.

[2] J.J. Bednarski, B.P. Sleckman, Lymphocyte development: integration of DNA damage response signaling, Adv. Immunol. 116 (2012) 175-204.

[3] P.A. Jeggo, J.A. Downs, Roles of chromatin remodellers in DNA double strand break repair, Exp. Cell Res. 329 (2014) 69-77.

[4] J.R. Walker, R.A. Corpina, J. Goldberg, Structure of the Ku heterodimer bound to DNA and its implications for double-strand break repair, Nature 412 (2001) 607-614.

[5] J.M. Jones, M. Gellert, W. Yang, A Ku bridge over broken DNA, Structure 9 (2001) 881-884

[6] S.A. Nick McElhinny, C.M. Snowden, J. McCarville, D.A. Ramsden, Ku recruits the XRCC4-ligase IV complex to DNA ends, Mol. Cell. Biol. 20 (2000) 2996-3003.

[7] M.L. Nicolette, K. Lee, Z. Guo, M. Rani, J.M. Chow, S.E. Lee, T.T. Paull, Mre11-Rad50-Xrs2 and Sae2 promote 5' strand resection of DNA double-strand breaks, Nat. Struct. Mol. Biol. 17 (2010) 1478-1485.

[8] H. Niu, W.-H. Chung, Z. Zhu, Y. Kwon, W. Zhao, P. Chi, R. Prakash, C. Seong, D. Liu, L. Lu, Mechanism of the ATP-dependent DNA end-resection machinery from Saccharomyces cerevisiae, Nature 467 (2010) 108-111.

[9] Z. Liang, S. Sunder, S. Nallasivam, T.E. Wilson, Overhang polarity of chromosomal double-strand breaks impacts kinetics and fidelity of yeast non-homologous end joining, Nucleic Acids Res. 44 (2016) 2769-2781.

[10] P. Cejka, S.C. Kowalczykowski, The full-length Saccharomyces cerevisiae Sgs1 protein is a vigorous DNA helicase that preferentially unwinds holliday junctions, J. Biol. Chem. 285 (2010) 8290-8301.

[11] A. Shibata, D. Moiani, A.S. Arvai, J. Perry, S.M. Harding, M.M. Genois, R. Maity, S. van Rossum-Fikkert, A. Kertokalio, F. Romoli, A. Ismail, E. Ismalaj, E. Petricci, M.J. Neale, R.G. Bristow, J.Y. Masson, C. Wyman, P.A. Jeggo, J.A. Tainer, DNA double-strand break repair pathway choice is directed by distinct MRE11 nuclease activities, Mol. Cell 53 (2014) 7-18.

[12] E. Cannavo, P. Cejka, Sae2 promotes dsDNA endonuclease activity within Mre11-Rad50-Xrs2 to resect DNA breaks, Nature 514 (2014) 122-125.

[13] E.P. Mimitou, L.S. Symington, Ku prevents Exo1 and Sgs1-dependent resection of DNA ends in the absence of a functional MRX complex or Sae2, EMBO J. 29 (2010) 3358-3369.

[14] S.J. Boulton, S.P. Jackson, Identification of a Saccharomyces cerevisiae Ku80 homologue: roles in DNA double strand break rejoining and in telomeric maintenance, Nucleic Acids Res. 24 (1996) 4639-4648.

[15] P. Frit, N. Barboule, Y. Yuan, D. Gomez, P. Calsou, Alternative end-joining pathway(s): bricolage at DNA breaks, DNA Repair (Amst.) 17 (2014) 81-97.

[16] A. Xie, A. Kwok, R. Scully, Role of mammalian Mre11 in classical and alternative nonhomologous end joining, Nat. Struct. Mol. Biol. 16 (2009) $814-818$.

[17] M. Lee-Theilen, A.J. Matthews, D. Kelly, S. Zheng, J. Chaudhuri, CtIP promotes microhomology-mediated alternative end joining during class-switch recombination, Nat. Struct. Mol. Biol. 18 (2011) 75-79.

[18] L.N. Truong, Y. Li, L.Z. Shi, P.Y. Hwang, J. He, H. Wang, N. Razavian, M.W. Berns, $\mathrm{X} . \mathrm{Wu}, \mathrm{Microhomology-mediated} \mathrm{End} \mathrm{Joining} \mathrm{and} \mathrm{Homologous}$ Recombination share the initial end resection step to repair DNA double-strand breaks in mammalian cells, Proc. Natl. Acad. Sci. U. S. A. 110 (2013) 7720-7725

[19] D.D. Villarreal, K. Lee, A. Deem, E.Y. Shim, A. Malkova, S.E. Lee, Microhomology directs diverse DNA break repair pathways and chromosomal translocations, PLoS Genet. 8 (2012) e1003026.

[20] A. Panday, L. Xiao, A. Grove, Yeast high mobility group protein HMO1 stabilizes chromatin and is evicted during repair of DNA double strand breaks, Nucleic Acids Res. 43 (2015) 5759-5770.

[21] A. Panday, A. Grove, The high mobility group protein HMO1 functions as a linker histone in yeast, Epigenet. Chromatin 9 (2016) 1.

[22] A. Panday, A. Grove, Yeast HMO1: linker histone reinvented, Microbiol. Mol Biol. Rev. 81 (2017) e00037-00016.

[23] D.B. Hall, J.T. Wade, K. Struhl, An HMG protein Hmo1, associates with promoters of many ribosomal protein genes and throughout the rRNA gene locus in Saccharomyces cerevisiae, Mol. Cell. Biol. 26 (2006) 3672-3679.

[24] M. Wittner, S. Hamperl, U. Stockl, W. Seufert, H. Tschochner, P. Milkereit, J. Griesenbeck, Establishment and maintenance of alternative chromatin states at a multicopy gene locus, Cell 145 (2011) 543-554

[25] L. Xiao, E. Kamau, D. Donze, A. Grove, Expression of yeast high mobility group protein HMO1 is regulated by TOR signaling, Gene 489 (2011) 55-62. 
[26] L. Yu, M.R. Volkert, Differential requirement for SUB1 in chromosomal and plasmid double-strand DNA break repair, PLoS One 8 (2013) e58015.

[27] L. Xiao, A.M. Williams, A. Grove, The C-terminal domain of yeast high mobility group protein HMO1 mediates lateral protein accretion and in-phase DNA bending, Biochemistry 49 (2010) 4051-4059.

[28] E. Kamau, K.T. Bauerle, A. Grove, The Saccharomyces cerevisiae high mobility group box protein HMO1 contains two functional DNA binding domains, J. Biol. Chem. 279 (2004) 55234-55240.

[29] S.J. Boulton, S.P. Jackson, Saccharomyces cerevisiae Ku70 potentiates illegitimate DNA double-strand break repair and serves as a barrier to error-prone DNA repair pathways, EMBO J. 15 (1996) 5093-5103.

[30] J.W. Westmoreland, J.A. Summers, C.L. Holland, M.A. Resnick, L.K. Lewis, v Blunt-ended DNA double-strand breaks induced by endonucleases PvuII and EcoRV are poor substrates for repair in Saccharomyces cerevisiae, DNA Repair (Amst.) 9 (2010) 617-626.

[31] M. Tsabar, V.V. Eapen, J.M. Mason, G. Memisoglu, D.P. Waterman, M.J. Long, D.K. Bishop, J.E. Haber, Caffeine impairs resection during DNA break repair by reducing the levels of nucleases Sae2 and Dna2, Nucleic Acids Res. 43 (2015) 6889-6901.
[32] N. Sanvisens, R. de Llanos, S. Puig, Function and regulation of yeast ribonucleotide reductase: cell cycle, genotoxic stress, and iron bioavailability, Biomed. J. 36 (2013) 51-58.

[33] M. Huang, Z. Zhou, S.J. Elledge, The DNA replication and damage checkpoint pathways induce transcription by inhibition of the Crt1 repressor, Cell 94 (1998) 595-605

[34] J. Rouse, S.P. Jackson, Lcd1p recruits Mec1p to DNA lesions in vitro and in vivo, Mol. Cell 9 (2002) 857-869.

[35] P. Langerak, E. Mejia-Ramirez, O. Limbo, P. Russell, v Release of Ku and MRN from DNA ends by Mre11 nuclease activity and Ctp1 is required for homologous recombination repair of double-strand breaks, PLoS Genet. 7 (2011) e1002271.

[36] K. Lee, S.E. Lee, Saccharomyces cerevisiae Sae2- and Tel1-dependent single-strand DNA formation at DNA break promotes microhomology-mediated end joining, Genetics 176 (2007) 2003-2014.

[37] K.T. Bauerle, E. Kamau, A. Grove, Interactions between N- and C-terminal domains of the Saccharomyces cerevisiae high-mobility group protein HMO1 are required for DNA bending, Biochemistry 45 (2006) 3635-3645. 